\title{
Surface forces and wetting features in drops and capillaries
}

\author{
M. G. Velarde ${ }^{1}$ \& V. M. Starov ${ }^{2}$ \\ ${ }^{I}$ Instituto Pluridisciplinar, Universidad Complutense de Madrid, Spain \\ ${ }^{2}$ Department of Chemical Engineering, Loughborough University, UK
}

\begin{abstract}
Using the DLVO (Derjaguin, Landau, Verwey, Overbeek) theory, which accounts for quantum mechanics and electrostatics at the macroscopic level, the thermodynamic expressions for (thermodynamic) equilibrium contact angles of drops on solid substrates and menisci in solid wall capillaries are, operationally and unambiguously, expressed in terms of the corresponding Derjaguin pressure. The latter's $S$-shape is responsible for microdrops and other phenomena appearing on flat solid substrates.
\end{abstract}

Keywords: DLVO, Derjaguin pressure, surface forces, wetting, drops, capillaries.

\section{Introduction: mechanics versus thermodynamics}

In the study of the equilibrium of a sessile drop partially wetting a smooth, homogeneous, flat solid substrate, there have been numerous attempts to deduce the Young equation for the equilibrium contact angle, $\theta,[1-3]$

$$
\cos \theta=\frac{\gamma_{s v}^{0}-\gamma_{s l}}{\gamma},
$$

where $\gamma_{s v}^{0}, \gamma_{s l}, \gamma$ are solid-vapor (of a bare solid surface), solid-liquid and liquid-vapor interfacial tensions, respectively. As reflected in most textbooks and scientific publications, traditional analyses use arguments either from thermodynamics or from mechanics, always insisting on eqn. (1) defining an "equilibrium" condition. As stated, eqn. (1) defines a contact angle, $\theta$, that does 


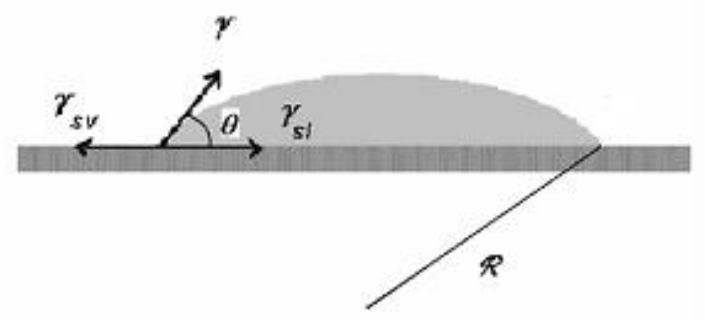

Figure 1: A sessile drop on a solid surface in apparent mechanical equilibrium. As shown, the angle $\theta$ defines, following Young, the mechanical equilibrium of tangential "forces".
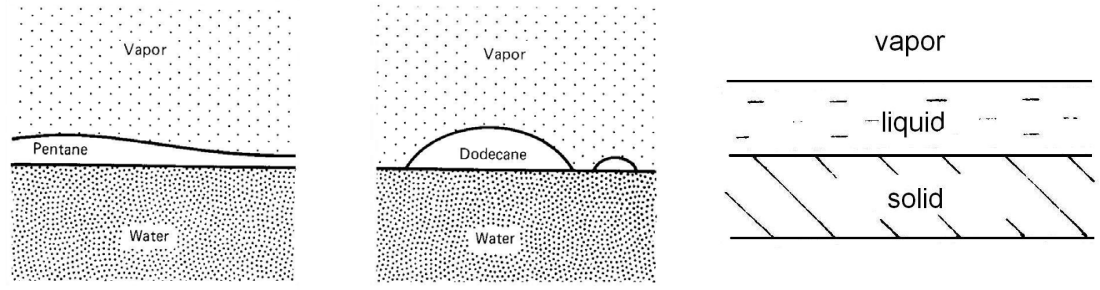

Figure 2: Wetting (left figure) and mostly non-wetting (center figure) liquid films and the "ideal" complete wetting case (figure on the right).

not depend on the vapor pressure in the ambient air or on the volume of the drop or on its radius of curvature, $\mathfrak{R}$. To be also noted is that neither $\gamma_{s v}^{0}$ nor $\gamma_{s l}$ can be measured [2].

However, to a useful first approximation, we have the Kelvin equation [2]

$$
P_{e}=\frac{2 \gamma}{\Re}=\frac{R T}{v_{m}} \ln \frac{p}{p_{s}},
$$

where $v_{m}$ is the molar volume of the liquid, $p_{s}$ is the pressure of the saturated vapor at the temperature $T, R$ is the universal gas constant, $p$ is the vapor pressure that is at equilibrium with the liquid in the drop, and $P_{e}$ is the excess pressure inside the drop. As the right hand side of eqn. (2) must be positive the vapor pressure, $p$, must be above the pressure of the saturated vapor $p_{s}$ (corresponding to a flat surface, $R \rightarrow \infty$ ). Hence the drop can be at equilibrium with oversaturated vapor pressure only. Thus, eqn. (2) determines a single radius of curvature of the liquid drop for each given value of oversaturated vapor pressure, $p$. Accordingly, the contact angle determined by eqn. (1) does not correspond to thermodynamic equilibrium. Note that, with oversaturation, the equilibration process demands quite long time intervals (on occasion up to hours). Surely most of the published data on measured contact angles actually 


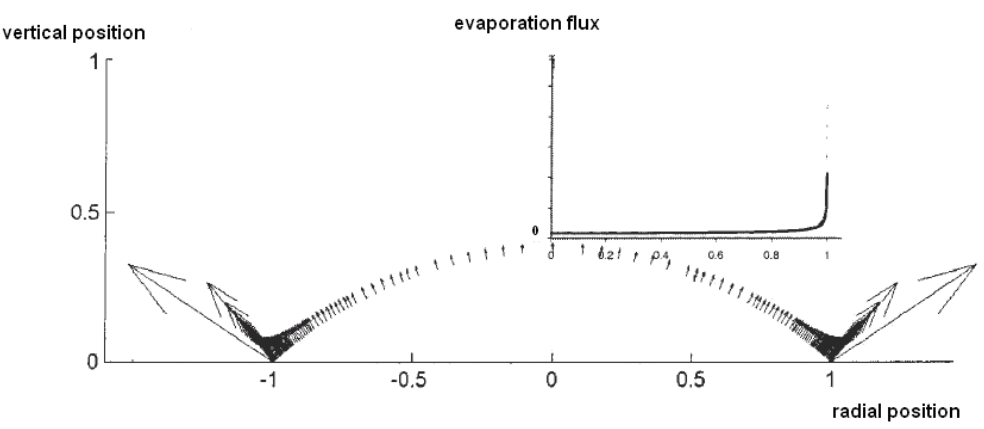

Figure 3: $\quad$ Evaporation flux along a sessile drop surface.

correspond to so-called (static) advancing contact angles, different from the true equilibrium value $[2,4,5]$.

If eqn. (1) refers to mechanical equilibrium then, disregarding gravity effects, the drop is bound to retain a spherical shape down to the contact with the solid substrate, that is, down to the three-phase contact line where the thickness of the liquid vanishes. To be recalled is that a displacement of the three-phase contact line through the solid substrate as the liquid wets it is hard to explain. Indeed, according to standard hydrodynamics, the tangential friction force at the threephase contact line diverges to infinite value. Another difficulty with eqn. (1), as it stands, is that evaporation seems to take place most vigorously in the vicinity of such three-phase contact line (fig. 2). This means that thermodynamic equilibrium is violated mostly just in the vicinity of the three-phase contact line $[6,7]$.

\section{DLVO theory drops and capillaries}

Starting with the work of Frumkin, Derjaguin and Landau, and Verwey and Overbeek, and Casimir, in the mid-XXth century, a useful approach (DLVO theory) to the problems raised by eqn. (1) [8-10] has been developed, thus leading to a sound thermodynamic derivation of the "true" equilibrium contact angle [4]. From the work of those authors it became clear that as the three-phase contact line belongs to the hundred nano-meter range then at such distances between two surfaces quantum mechanics and electrostatics intervene. When all their integrated molecular interactions are considered, this led to the introduction of so-called "surface forces" and the definition of a new form of pressure of significance in the study of thin liquid films and, in general, when two surfaces approach each other in vacuum or otherwise, as the "ideal" case illustrated in fig. 2. Originally, it was denoted as "disjoining" pressure though depending on the confronted surfaces and the medium separating them it could be either disjoining or conjoining. We shall call it Derjaguin pressure.

How much the action of Derjaguin pressure affects the liquid profile eventually deviating it from a spherical shape when approaching the three-phase 


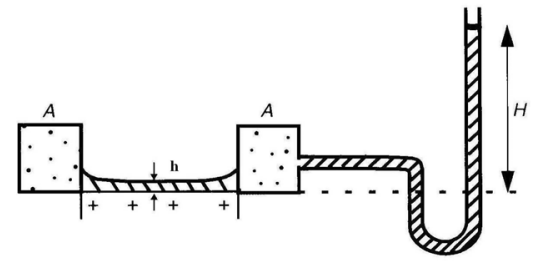

Figure 4: Measuring Derjaguin pressure (as proposed by Derjaguin, Churaev and Sheludko) using a porous container $A$ : $\Pi(h)=-\rho g H$ ( $H$ may be positive or negative).
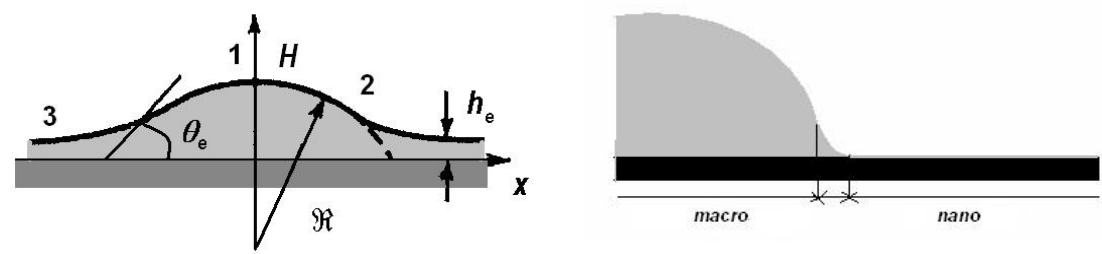

Figure 5: Exaggerated view (as $H>>h_{e}$ ) of a liquid drop partially wetting a solid substrate. $\mathfrak{R}$ is the radius of the "spherical" cap. In the left figure the angle $\theta_{e}$ can be considered as the contact angle at (thermodynamic) equilibrium corresponding (following Gibbs) to the minimum excess free energy of the system relative to the homogeneous flat thin liquid film. The right figure illustrates the spherical macro-region, the adsorbed nano-metric liquid film and the transition region.

contact line? How the (thermodynamic) equilibrium contact angle can be defined? These questions can be answered by considering the combined action of Laplace capillary ("surface tension") pressure and Derjaguin ("surface forces") pressure acting along the overall drop profile. The Laplace pressure is [2-5]

$$
p_{l}-p_{a}=\frac{2 \gamma}{\mathfrak{R}} \quad \text { or otherwise } \quad \frac{\gamma h^{\prime \prime}}{\left(1+h^{\prime 2}\right)^{3 / 2}}=P_{e},
$$

where for simplicity in two-dimensional geometry (a cylindrical drop) we have provided the value of $\mathfrak{R}$ in terms of the function $h$ defining the drop profile. $P_{e}=p_{a}-p_{l}$ is negative for a drop and positive for a capillary.

To carry on the proposed task we must consider that at thermodynamic equilibrium the liquid in the drop must be at equilibrium with its own vapor (recall Kelvin equation), and also at equilibrium with the solid, both under the drop and around it, and the vapor must be at equilibrium with the solid substrate. The latter demands the existence of an adsorbed liquid film (of thickness $h_{e}$ ) on the solid surface as illustrated in fig. 5. A similar figure can be provided for a capillary with, however, opposite curvature $[2,4,5]$. 
Let us assume that we can maintain the oversaturated vapor condition over the solid substrate long enough until thermodynamic equilibrium is reached. Then the liquid molecules in the vapor are in equilibrium with the liquid molecules in the drop and with those in the adsorbed film on the solid surface. With either partial or complete wetting conditions, the presence of an adsorbed liquid layer on the solid substrate demands consideration of a new surface tension $\gamma_{s v h_{e}}$ of lower values than the surface tension of the bare solid surface, $\gamma_{s v}^{0}$. In view of the above, the expression for the excess free energy of the overall liquid layer over the solid substrate contains the free energies due to the spherical part of the drop and to the adsorbed liquid film with the transition zone:

$$
\Phi_{f}=\gamma+P_{e} h_{e}+\int_{h_{e}}^{\infty} \Pi(h) d h+\gamma_{s l}-\gamma_{s v}^{0} .
$$

As earlier noted, $P_{e}$ is a given Laplace excess capillary pressure and $\Pi(h)$ is the Derjaguin pressure for a given temperature.

We can rewrite the expression (4) as $\Phi_{f}=\gamma_{s v h_{e}}-\gamma_{s v}^{0}$, by introducing

$$
\gamma_{s v h_{e}}=\gamma+P_{e} h_{e}+\int_{h_{e}}^{\infty} \Pi(h) d h+\gamma_{s l},
$$

as the interfacial tension (which is an excess free energy) of the solid substrate covered with the adsorbed liquid film. Then, inserting $\gamma_{s v h_{e}}$ rather than $\gamma_{s v}^{0}$ in Young equation (1) we get

$$
\cos \theta_{e}=\frac{\gamma_{s v h_{e}}-\gamma_{s l}}{\gamma}
$$

thus defining a contact angle at thermodynamic equilibrium. Consequently,

$$
\cos \theta_{e}=\frac{\gamma+P_{e} h_{e}+\int_{h_{e}}^{\infty} \Pi(h) d h}{\gamma} \approx 1+\frac{1}{\gamma} \int_{h_{e}}^{\infty} \Pi(h) d h,
$$

if recalling the smallness of $h_{e}\left(H>>h_{e}\right)$ we neglect the term $P_{e} h_{e} / \gamma$. The latter equation is the Derjaguin-Frumkin equation for the (thermodynamic) equilibrium contact angle. It provides an operational definition relative to eqn. (1) which is conceptually unacceptable.

The argument is formally valid for both a drop and a capillary. As $-1<\cos \theta_{e}<1$, in the case of partial wetting (water and aqueous solutions over most solid substrates) the integral in the right hand side of eqn. (7) must be negative $\int_{h_{e}}^{\infty} \Pi(h) d h<0$, and hence for partial wetting $S_{-}>S_{+}$(actually, this condition defines partial wetting). Note that there are an infinite number of equilibrium contact angles for a drop on a solid substrate (each of them corresponds to a different value of the oversaturated vapor pressure in the 

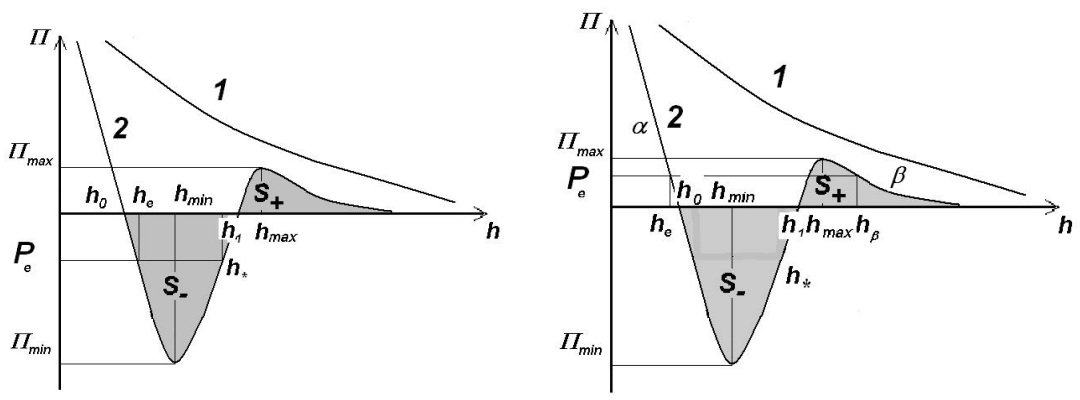

Figure 6: Derjaguin pressures, $\Pi(h)$, interpreted as isotherms corresponding to the cases of complete wetting (1) and partial wetting (2). The left figure is for drops and the right figure is for capillaries. In the latter the liquid film thicknesses, $h_{e}, h_{\beta}$ and $h_{*}$, are thin flat films that for a given non-vanishing value of $P_{e}$ could coexist at equilibrium with the central part of the meniscus in a capillary when $P_{e}<\Pi_{\max }$. However, only the first two are thermodynamically stable as $\frac{d \Pi(h)}{d h}<0$ for either $h_{e}$ or $h_{\beta}$. Hence $h_{*}$ is unstable. For a drop as $P_{e}$ is negative the left figure shows that there is only $h_{e}$ and $h_{*}$, stable and unstable thicknesses, respectively. The values $h_{0}$ and $h_{1}$ denote the zeros of $\Pi(h)$ with no other meaning. For $h_{\min }$ and $h_{\max }$ see the main text.

surrounding air) but there is a single, uniquely defined equilibrium contact angle of the liquid meniscus in a partially wetted solid capillary for any undersaturated vapor pressure.

For completeness let us now focus on the capillary case. There are three solutions of equation $\Pi\left(h_{e}\right)=P_{e}>0$ (fig. 6, right part, curve 2). Only those satisfying $\frac{d \Pi\left(h_{e}\right)}{d h}<0$ are thermodynamically stable. The thinnest film $h_{e}$ belongs to the so-called $\alpha$-branch of the Derjaguin isotherm and it is absolutely stable. The thickest film, $h_{\beta}$, is a metastable solution, with a barrier $h_{\max }$, and belongs to the so-called $\beta$-branch. Then $h_{*}$ corresponds to an unstable equilibrium flat film. On the surface of thin capillaries only equilibrium $\alpha$-films are observed if $P_{e}>\Pi_{\max }$ where $\Pi_{\max }$ is the stability limit of $\beta$-films (fig. 6). This is the case for narrow enough capillaries. However, if $P_{e}<\Pi_{\max }$, the formation of metastable $\beta$-films is possible, and it has been observed experimentally. 
The $\alpha$-film is like an adsorbing film while the $\beta$-film is called a wetting film as it can be obtained by draining a thicker liquid layer. Generally, the free energy of $\alpha$-films is lower than the free energy of $\beta$-films, ruling out spontaneous $\alpha \rightarrow \beta$ transitions. Typical $\alpha$-films are the equilibrium films occurring when a hydrophilic surface is wetted with water. On the other hand, if more of the same liquid is added once the $\alpha$-film is formed, disregarding gravity, it will not spread but rather it will form drops over the $\alpha$-film (the fact that a liquid does not spread on itself is called autophobicity). Note that $\alpha$ or $\beta$-films may not exhibit interference colors when irradiated with white light and when they appear black they are usually called black films. In fact they do have destructive interference as two light rays/waves one reflected from the upper surface of the film and the other from the opposite surface, travel about the same path length but the second comes from the liquid film phase-shifted half a wavelength. These black liquid films were studied by Newton and it is customary to call the $\alpha$-black film, a Newton black film, whereas the $\beta$-black film is called common black film. Aqueous soap solutions easily permit visualizing this phenomenon. Indeed, the transition from a thick film to a black film is easily demonstrated by allowing a vertical soap film supported on a horizontal frame to drain. Initially the whole film exhibits interference colors when illuminated, and then a dark boundary, separated from the colored area by a silvery band appears at the top and moves downward. This then divides into two areas occupied by black films. Note that a bi-layer of soap molecules is just a few nano-meters thick.

\section{Non-flat equilibrium liquid films on flat surfaces: general thermodynamic approach}

In a thick liquid film if its surface is curved or uneven, the Derjaguin and the Laplace (capillary) pressures acting together permit stable non-flat equilibrium shapes. Dictated by the $S$-shaped Derjaguin pressure, in the case of partial wetting, micro-drops, micro-depressions and spatially periodic (wavy) films could exist on homogeneous, flat solid substrates [11].

Let us insist in that eqn. (7) also shows that the equilibrium contact angle is completely determined by the shape of the disjoining pressure in the case of molecularly smooth solid substrates. There is no doubt that the surface roughness influences the apparent value of the contact angle. However, the roughness cannot result in a transition from non-wetting to partial wetting, or from partial wetting to complete wetting. The possible existence of non-flat equilibrium liquid films points to a scenario of rupture of "thick" metastable $\beta$-films and their transition to absolutely stable $\alpha$-films.

Neglecting the action of gravity and, for simplicity, restricting again consideration to a two-dimensional capillary, the excess free energy of the system can be written as 


$$
\Phi=\int\left\{\gamma\left(\sqrt{1+h^{\prime 2}}-1\right)+P_{e}\left(h-h_{e}\right)+\int_{h}^{\infty} \Pi(h) d h-\int_{h_{e}}^{\infty} \Pi(h)\right\} d x,
$$

where the coordinate $x$ is taken along the flat solid surface of the wetted capillary. Such excess free energy is the excess over the energy of the same flat surface covered by a thermodynamically stable equilibrium $\alpha$-film. Although such reference state results in an additive constant in expression (8), it is needed to study the liquid profile in a vicinity of the apparent three-phase contact line. Any liquid profile, $h(x)$, which gives the minimum value to the excess free energy, $\Phi$, describes an equilibrium configuration. For a minimum we must have: (i) $\delta \Phi=0$; (ii) if $f=\gamma\left(\sqrt{1+h^{\prime 2}}-1\right)+P_{e}\left(h-h_{e}\right)+\int_{h}^{\infty} \Pi(h) d h-\int_{h_{e}}^{\infty} \Pi(h)$, then $\frac{\partial^{2} f}{\partial h^{\prime 2}}>0$; (iii) as the solution is a curve, $h(x)$, the problem is in functional space and a secondary variational principle is needed [12]. This leads to a Jacobi equation $\frac{d}{d x} \frac{\gamma u^{\prime}}{\left(1+h^{\prime 2}\right)^{3 / 2}}+\frac{d \Pi(h)}{d h} u=0$ whose solution cannot vanish when the unknown, $x$, is inside the region under consideration, except at the boundaries of the integration; and (iv) at the three-phase contact line, a smooth transition must occur from a non-flat liquid profile to a flat liquid film (transversality condition): $\left[f-h^{\prime} \frac{\partial f}{\partial h^{\prime}}\right]_{x=\Re}=0$. The actual three-phase contact line, virtually located at the border of $\mathfrak{R}$ in fig. 5, corresponds to the intersection of the evolving liquid profile with the equilibrium liquid film of thickness, $h_{e}$, and not to the intersection with the solid substrate. Then $\left[\left(\sqrt{1+h^{\prime 2}}-1\right)-\frac{h^{\prime 2}}{\sqrt{1+h^{\prime 2}}}\right]_{x=\Re}=0$, or $\left[\frac{1}{\sqrt{1+h^{\prime 2}}}\right]_{x=\Re}=1$. The latter and hence the mentioned smooth transition occurs when $\left(h^{\prime}\right)_{x=\Re}=0$, or $h^{\prime}\left(h_{e}\right)=0$, hence $h^{\prime} \rightarrow 0, x \rightarrow \infty$, where $x \rightarrow \infty$ means that it "tends to the end of the transition zone".

The first condition (i) yields

$$
\frac{\gamma h^{\prime \prime}}{\left(1+h^{\prime 2}\right)^{3 / 2}}+\Pi(h)=P_{e}
$$

which is the Laplace-Derjaguin equation. Indeed, the first term in the left hand side of eqn. (9) corresponds to the Laplace pressure and the second term to the Derjaguin pressure. Note that for a flat film the first term of the left hand side vanishes and hence $\Pi(h)=P_{e}$. If the thickness of the liquid is beyond the range of the surface forces action (above hundred nm) then eqn. (9) describes either a 
flat liquid surface $\left(P_{e}=0\right)$, or a spherical drop profile $\left(P_{e}<0\right)$, or a curved meniscus profile $\left(P_{e}>0\right)$. The second condition (ii) is always satisfied.

Direct differentiation of eqn. (9) yields $\frac{d}{d x} \frac{\gamma h^{\prime \prime}}{\left(1+h^{\prime 2}\right)^{3 / 2}}+\frac{d \Pi(h)}{d h} \frac{d h}{d x}=0$.

Accordingly, the solution of the Jacobi equation is $u=\operatorname{const} h^{\prime}$. Hence, there is stability if $h^{\prime}\left(x_{0}\right)=0$ with $x_{0}$ different from the boundary points; otherwise the system is unstable.

Integrating equation (9) we have: $\frac{1}{\sqrt{1+h^{\prime 2}}}=\frac{C-P_{e} h-\int_{h}^{\infty} \Pi(h) d h}{\gamma}$, where $C$ is an integration constant. Needless to say the right hand side must be positive. In the case of a meniscus in a flat capillary the integration constant, $C$, is determined from the condition that at the capillary center: $h^{\prime}(H)=-\infty$, which gives $C=P_{e} H$, where here $H$ is the half-width of the capillary. In the case of equilibrium drops the constant should be chosen using the condition that at the drop apex, $h=H: h^{\prime}(H)=0$, which results in $C=\gamma+P_{e} H$. An alternative way of getting the integration constant $C$ is using the transversality condition.

A profile of the transition zone between a non-flat meniscus in a flat capillary and a thin $\alpha$-film ahead of it in the case of partial wetting is depicted in fig. 7 (to be compared with fig. 6, right part, curve 2, for a flat meniscus). In the transition zone both Laplace and Derjaguin pressures act simultaneously. It appears that the liquid profile is not of uniform concavity from the vapor side, but changes curvature inside the transition zone.

In the transition zone (fig. 7, right part) all thicknesses appear from the very thick, outside the range of the Derjaguin pressure action, to the thin $\alpha$-films.

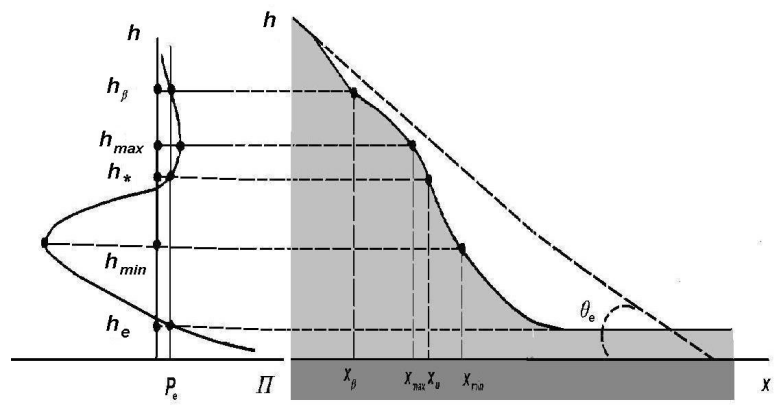

Figure 7: Partial wetting of a capillary. Enlarged view of the liquid profile inside the transition zone in "thick" capillaries. $S$-shaped disjoining pressure isotherm (left side) and the liquid profile in the transition zone (right side). Here $h_{e}$ corresponds to the stable "thin" $\alpha$-film, and $h_{\beta}$ to "thick" $\mathrm{t}$-films as defined by $\Pi(h)=P_{e}>0$. 
Then the Jacobi equation condition (iii) above must be used rather than the stability condition (4) used in the case of flat films. Indeed, the transition zone is stable if $h^{\prime}(x)$ does not vanish inside it. Such peculiar shape of the transition zone, where both the capillary pressure and the disjoining pressure are equally important, suggests looking for stable solutions other than flat liquid films of constant thickness.

\section{Non-flat equilibrium films. Microdrops: the case $P_{e}>0$ (undersaturation conditions)}

Let us consider the possible existence of microdrops, i.e., drops with an apex height in the range of influence of the Derjaguin pressure [4, 11]. In this case the drop does not have a spherical part even at its apex, because its shape is everywhere distorted by the action of the surface forces. The transversality condition at $h=h_{e}$ gives $h^{\prime}\left(h_{e}\right)=0$, and hence the drop edge approaches the equilibrium film of thickness $h_{e}$ on the solid surface at vanishing microscopic contact angle. This permits to fix the earlier mentioned integration constant $C=\gamma+P_{e} h_{e}+\int_{h_{e}}^{\infty} \Pi(h) d h$. Then, the microdrop profile (fig. 8; to be compared with fig. 6, left part, curve 2) follows from imposing $h^{\prime}=-\sqrt{\frac{\gamma^{2}}{[\gamma-L(h)]^{2}}-1}$,
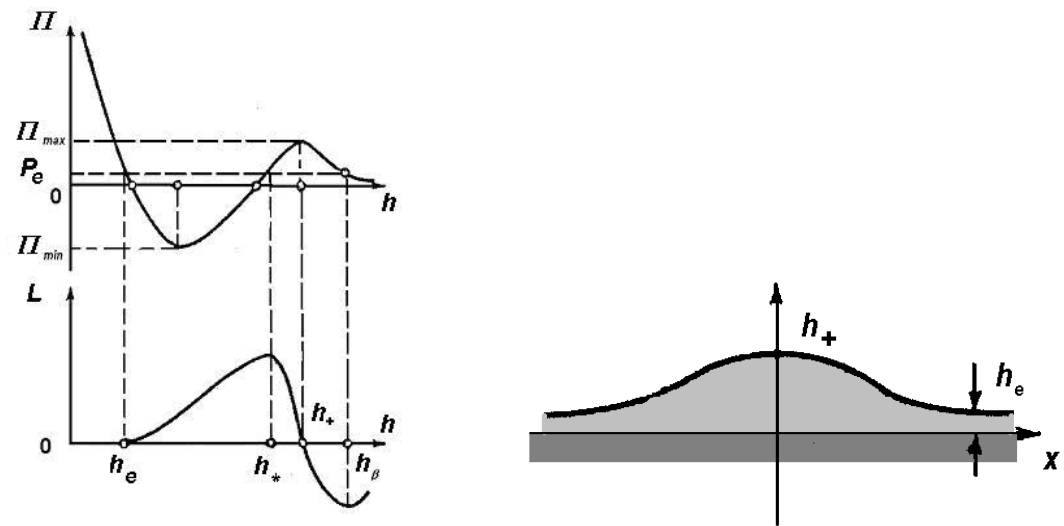

Figure 8: $\quad$ Microdrops. Left figure, upper part: $S$-shaped Derjaguin (pressure) isotherm; bottom part: $L(h)$ (see main text). The value $L\left(h_{+}\right)=0$ determines the drop apex, $h_{+}$. Right figure: profile of an equilibrium microdrop. The apex of the microdrop is in the range of the Derjaguin pressure action, and hence the drop is not spherical even at the drop apex. 
with $L(h)=P_{e}\left(h-h_{e}\right)-\int_{h_{e}}^{h} \Pi(h) d h \quad$ and $\quad 0 \leq L(h) \leq \gamma . \quad$ The first equality corresponds to vanishing derivative, $h^{\prime}$, while the second one corresponds to the infinite derivative.

Let $h_{+}$define the apex of the microdrop. The top part of the left fig. 8, shows the $S$-shaped dependence of the Derjaguin isotherm, $\Pi(h)$, while the bottom part shows the curve, $L(h)$, which is maximum or minimum when $P_{e}=\Pi(h)$. At the apex of the drop, when $h=h_{+}$, the first derivative must be zero, $h^{\prime}\left(h_{+}\right)=0$ and hence $L\left(h_{+}\right)=0$. Take the origin placed at the center of the microdrop which corresponds to its formation at undersaturation, $P_{e}>0$. In the range $0<P_{e}<\Pi_{\max }$ the equation $P_{e}=\Pi(h)$ has three roots (fig. 8, left part); the smallest corresponds to the equilibrium flat $\alpha$-film of thickness, $h_{e}$. For the existence of microdrops we must have: $h^{\prime \prime}<0$ at $h=h_{+}$and $h^{\prime \prime}>0$ as $h \rightarrow h_{e}$, hence $h_{*}<h_{+}<h_{\beta}$. Then $P_{e}\left(h_{+}-h_{e}\right)=\int_{h_{e}}^{h_{+}} \Pi(h) d h$, whose solution, $h_{+}$, must satisfy the inequality $h_{u}<h_{+}<h_{\beta}$ as illustrated in the bottom part of fig. 8, left part. As the left hand side is positive, so must be its right hand site. Hence, a sufficient condition for the existence of equilibrium microdrops is that $S_{-}<S_{+}$. This implies that the $S$-shaped Derjaguin (pressure) isotherm leads to an equilibrium contact angle of zero value. Consequently, microdrops at thermodynamic equilibrium are not possible if there is partial wetting, when $S_{-}>S_{+}$or with complete wetting, when the Derjaguin pressure decreases in a monotonic way, as for example with $\Pi(h)=A / h^{3}$.

\section{Concluding remarks}

Summarizing, we have shown that the thermodynamics of drops and liquid films with curved menisci involves, for a given temperature, both the Laplace (capillary) pressure and the Derjaguin (surface forces) pressure. Once the Derjaguin (pressure) isotherm is known we can define, operationally and unambiguously, what do we mean by complete wetting, partial wetting and nonwetting. For the partial wetting case, the $S$-shaped Derjaguin isotherm, determines "true" thermodynamic equilibrium contact angles for drops of capillaries. Such $S$-shaped form is also responsible of the possible existence of microdrops, and even for micro-depressions and equilibrium periodic films on flat solid substrates.

\section{Acknowledgement}

This research was supported by the EU under Grant MULTIFLOW, FP7-ITN2008-214919. 


\section{References}

[1] Young, T., An essay on the cohesion of fluids. Phil. Trans. Royal Soc. London, 95, pp. 65-87, 1805.

[2] Adamson, A.W. \& Gast, A.P., Physical Chemistry of Surfaces, $6^{\text {th }}$ ed., Willey: New York, 1997 (and references therein).

[3] de Gennes, P.G., Brochard-Wyart, F. \& Quéré, D., Capillarity and Wetting Phenomena. Drops, Bubbles, Pearls, Waves, Springer-Verlag: New York, 2004.

[4] Starov, V.M., Velarde, M.G. \& Radke, C.J., Wetting and Spreading Dynamics, Taylor \& Francis /CRC: New York, 2007.

[5] Starov, V.M. \& Velarde, M.G., Surface forces and wetting phenomena. J. Phys. Cond. Matter, 21, pp. 464121-1-11, 2009.

[6] Starov, V.M. \& Sefiane, K., On evaporation rate and interfacial temperature of volatile sessile drops. Colloids \& Surfaces A. Physicochem. Engng. Aspects, 333, pp. 170-174, 2009.

[7] Ajaev, V.S. Gambaryan-Roisman, T. \& Stephan, P., Static and dynamic contact angles of evaporating liquids on heated surfaces. J. Colloid Interface Sci., 342, pp. 550-558, 2010.

[8] Derjaguin, B.V., Churaev, N.V. \& Muller, V.M., Surface Forces, Consultants Bureau-Plenum Press: New York, 1987 (and references therein).

[9] Verwey, E.J.W. \& Overbeek, J.Th.G., Theory of the Stability of Lyophobic Colloids, Elsevier: Amsterdam, 1948.

[10] Israelachvili, J.N., Intermolecular and Surface Forces, $2^{\text {nd }}$ ed., Academic Press: London, 1991.

[11] Starov, V.M., Nonflat equilibrium liquid shapes on flat surfaces. J. Colloid Interface Sci., 269, pp. 432-441, 2004.

[12] Sagan, A., Introduction to the Calculus of Variations, Dover reprint: New York, chap. 7, 1992. 